\title{
Estrategias de vida y rutas de desarrollo de jóvenes rurales en Nicaragua: los casos en Muy Muy y en Matiguás
}

\section{Griet Steel y Carlos Sosa}

\section{Recibido: junio de 2011 / Aceptado: julio de 2011}

Existe una preocupación creciente en la industria del desarrollo sobre la situación de hombres y mujeres jóvenes creciendo en contextos de pobreza. Sin embargo, aún hay una escasez de investigación que dé cuenta de lo que ocurre en la vida diaria de los jóvenes y la forma en que éstos experimentan y tratan de manejar las situaciones de pobreza. Los estudios de juventud generalmente consideran a las personas jóvenes como pasivos receptáculos de las decisiones y políticas definidas por los adultos, y tienden a ignorar la capacidad que tienen las personas jóvenes de generar riqueza. En el presente artículo enfatizamos la necesidad de hacer investigación más profunda sobre las estrategias de vida de los jóvenes y su transición hacia la adultez. Sobre la base de estudios de caso de jóvenes rurales nicaragüenses, analizamos cómo los jóvenes de Muy Muy y Matiguás negocian sus estrategias de vida con sus padres, con la perspectiva de acumular recursos necesarios para lograr independizarse. En el desenvolvimiento de sus estrategias de vida reconocemos a los jóvenes como agentes activos del desarrollo de sus hogares y localidades. En esta medida, promovemos que las instituciones de desarrollo razonen sobre las iniciativas de los jóvenes en sus intervenciones de desarrollo. En este sentido, el presente estudio tiene la perspectiva no sólo de enriquecer el debate académico alrededor de las estrategias de vida de los jóvenes sino también de proveer insumos a las organizaciones que implementan estrategias de desarrollo con jóvenes en Nicaragua y la región.

Palabras clave: Jóvenes / Estrategias de vida / Desarrollo rural

\footnotetext{
* Instituto de Políticas y Gestión del Desarrollo (IOB), Universidad de Amberes, Amberes, Bélgica.

** Instituto de Investigación Aplicada y Promoción del Desarrollo Local, Nitlapan. Universidad Centroamericana, Edificio Nitlapan. Managua, Nicaragua. Tel. (505) 2278-0627 ext: 154, correo electrónico: soscarlos@gmail.com.
} 


\section{Introducción}

En los últimos años se observa una preocupación creciente en las agencias de desarrollo sobre la situación de hombres y mujeres jóvenes madurando en un contexto de pobreza y privaciones. Véase por ejemplo el "Programa de Acción Mundial para los Jóvenes” de las Naciones Unidas. Sin embargo, hay todavía pocos estudios académicos sobre la vida cotidiana de jóvenes marginalizados y la manera en que ellos viven y tratan de enfrentar situaciones de pobreza. La posición socioeconómica de las personas jóvenes y sus estrategias de vida no ha recibido mucha atención de los académicos, las políticas y las organizaciones de desarrollo (Ansell, 2004). Por consiguiente, hay una escasez de conocimiento académico y experiencia práctica sobre cómo se puede incorporar activamente a los jóvenes en programas de desarrollo y facilitar sus transiciones hacia la adultez.

Intentando contribuir a llenar esta laguna, este trabajo se enfoca en los jóvenes rurales de Muy Muy y Matiguás que viven en situaciones de pobreza. Para analizar las diversas estrategias de vida de estos jóvenes nos preguntamos cómo implementan y negocian sus estrategias de vida para sortear la pobreza y transitar hacia la adultez. Tratamos de encontrar repuestas siguiendo la forma en que los jóvenes buscan opciones agropecuarias y no agropecuarias, dentro y fuera de la comunidad, visualizando diferencias entre las experiencias de los hombres y de las mujeres. Este esfuerzo busca abrir la discusión alrededor del rol de los jóvenes en los procesos de desarrollo y la necesidad de analizar los medios y estrategias de vida de las personas jóvenes con el propósito de identificar y entender las rutas de desarrollo que toman estas personas para mejorar las intervenciones de desarrollo en el territorio. De esta forma queremos dar reconocimiento a la diferenciación generacional existente en las estrategias de los hogares y mostrar rutas similares y contrastantes entre las generaciones.

Este artículo se basa en un trabajo etnográfico realizado en 2010 en siete comunidades de Muy Muy y Matiguás, dos municipios del centro del país. De Muy Muy se escogió el territorio El Bálsamo ${ }^{1}$; y de Matiguás el territorio Pancasán ${ }^{2}$ (ver ilustración 1). En un equipo de dos investigadores implementamos siete talleres de investigación con 136 jóvenes. Estos jóvenes se encuentran entre los 20 y los 30 años y cuentan con cierto nivel de autonomía frente a sus padres, algunos forman su propia familia y estrategias de vida. Con ellas y ellos implementamos métodos que les permitieron expresarse fuera del formato convencional de preguntas y respuestas (Caputo, 1995). En los talleres hicimos ejercicios escritos donde pudieron expresar sus sueños y perspectivas. Organizamos mesas de discusión promoviendo el debate grupal sobre las oportunidades de los jóvenes en la comunidad. También se hicieron mapas cognitivos con grupos de mujeres y hombres, donde plasmaron los vínculos que establecen entre diferentes lugares importantes en su vida. Posteriormente entrevistamos a 19 mujeres y 11 hombres para juntar información detallada sobre sus estrategias de vida y la interacción con las de sus padres. En seis casos entrevistamos al hombre y a la mujer del hogar joven. El artículo se basa en 24 casos. Además

1 Compuesto por las comunidades de El Bálsamo 1 y El Bálsamo 2, El Dorado y La Luna.

2 Que contempla las comunidades de El Jobo, Pancasán y Sitio Histórico. 
experimentamos con la metodología de fotovoz (Collier \& Collier, 1996), con la que los entrevistados visualizaron la realidad socioeconómica local y sus aspiraciones.

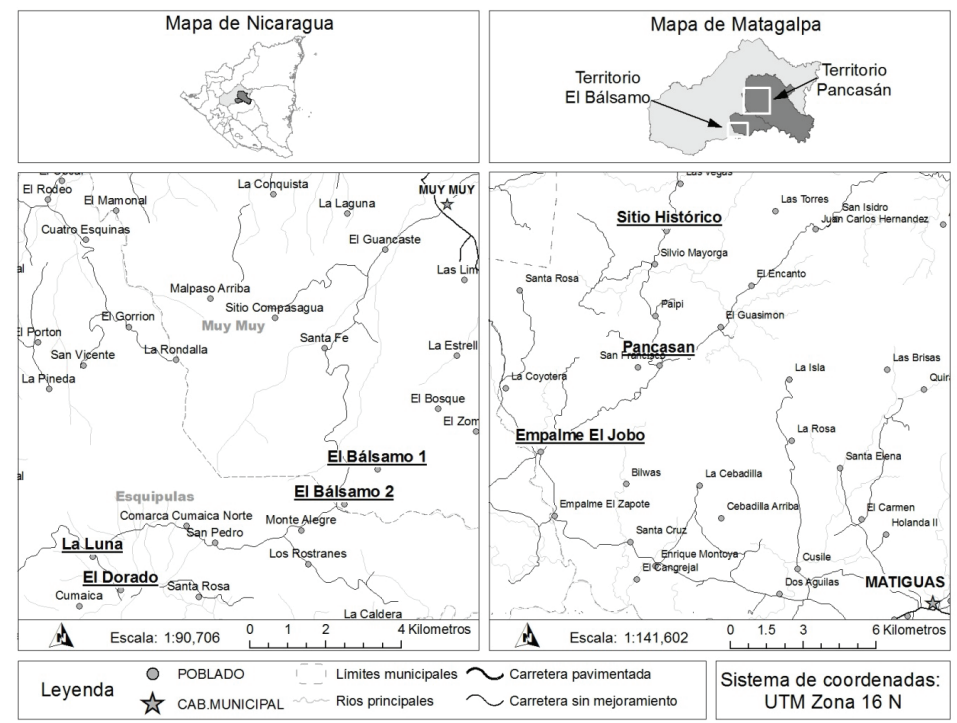

Ilustración 1. Mapas de los territorios de El Bálsamo y Pancasán

Fuente: Elaborado por Carlos Sosa, Nitlapan, 2011. Datos del CENAGRO (INIDE, 2001).

En el presente artículo abordaremos aspectos teóricos sobre los procesos de transición hacia la adultez, y revisaremos el aporte de los enfoques de medios de vida y la centralidad del actor al entendimiento de las estrategias de vida de jóvenes, contrastándolos con experiencias de desarrollo con jóvenes. Proveemos posteriormente información de contexto de los hogares estudiados para luego presentar tres tipos de estrategias de independencia: las estrategias integradas; las estrategias mediadas por los padres; y finalmente, las de mayor autonomía frente a los padres. Exploramos las características de cada tipo, sus oportunidades y también sus amenazas en el contexto en el que viven. En las conclusiones hacemos hincapié en entender los procesos de interdependencia para intentar dar pistas sobre cómo mejorar las acciones de desarrollo con jóvenes.

\section{Estrategias de vida de jóvenes y transiciones hacia la adultez}

Transitar hacia la adultez para muchos jóvenes en el mundo de hoy "es quizá más complejo y competitivo que en épocas anteriores” (Jeffrey \& Mcdowell, 2004). La desregulación laboral, la creciente concentración de la riqueza y la crisis económica global dificultan que los jóvenes empobrecidos puedan independizarse. Sin embargo, todavía hay pocos estudios que documentan las transiciones hacia la adultez, sobre todo en países en vías de desarrollo (exceptuando los estudios de Punch, 2002; Ansell, 2004; Van Blerk, 2008; y Crivello, 2010). Además, como Chant y Jones (2005, p. 196) expresan, los académicos y profesionales del desarrollo todavía ven la 
transición hacia la adultez como rutas unilineales, tal como de la educación hacia el trabajo, del trabajo hacia el alivio de la pobreza o de la niñez hacia la adultez.

En realidad la transición hacia la adultez se desenvuelve en múltiples procesos complejos en que se pueden observar interrelaciones entre la educación y el trabajo o entre las estrategias de vida de los jóvenes y las de sus padres. En este sentido, no son transiciones en las que todos los roles del adulto son asumidos al mismo tiempo (Lloyd, 2005, p. 27). O como Punch (2002) lo expresa, no se puede hablar de una transición de niño dependiente hacia adulto independiente porque en países en vías de desarrollo, la gente joven negocia y renegocia sus estrategias y responsabilidades todo el tiempo con sus padres y otros familiares. En este sentido, ella propone el concepto de 'interdependencia' para poder analizar las relaciones entre jóvenes y adultos en áreas rurales de países en vías de desarrollo y sus transiciones hacia la adultez.

Bajo esta perspectiva es necesario pasar de un enfoque pasivo a uno activo sobre el papel que juegan los jóvenes en la sociedad. Con frecuencia, los estudios sobre juventud todavía ven a 'la juventud' como receptora de decisiones y políticas definidas por los adultos, aparentemente ignorándose la capacidad que tienen los jóvenes de generar ingresos por su propia cuenta. Contrariamente a este enfoque pasivo, de los nuevos estudios sociales sobre niñez y trabajo infantil ha surgido el enfoque en la agencia, el cual remarca la construcción social de la niñez (James \& Prout, 1990). Este enfoque considera a las personas jóvenes como actores sociales con derecho a ser respetados en su propia manera (Bucholtz, 2002; Caputo, 1995; James \& Prout, 1990; Liebel, 2003; Valentine, 1996).

La incorporación de un enfoque en el actor a los estudios de las estrategias de vida de jóvenes pobres da luces sobre la diversidad y multidimensionalidad de sus vidas. Dicha incorporación integra la conceptualización dinámica, multidimensional y participativa de la pobreza, tal como es desarrollada en el enfoque de medios de vida (Chambers, 1995; Carney, 1998; Kaag et al., 2004). Este enfoque centrado en la gente enfatiza que los jóvenes construyen sus estrategias de vida con una perspectiva socioeconómica y espacial más amplia. Los jóvenes pueden estar limitados por el contexto estructural de un ambiente de pobreza institucionalizada (Bastiaensen, De Herdt, \& D'Exelle, 2005) y las expectativas culturales de responsabilidades familiares. No obstante, tienen la habilidad de actuar dentro y en medio de estas restricciones estructurales (Giddens, 1984; Long, 2001) y usar, adaptar, interpretar e incluso cambiar, sus posibilidades y limitaciones de acuerdo a sus propias preferencias y oportunidades. Por tanto, sus transiciones hacia la adultez deberían ser interpretadas desde una perspectiva de 'interdependencia negociada' en que por un lado se reconocen las restricciones estructurales y culturales -como las expectativas de responsabilidades familiares- pero por otro lado no se niega la agencia del joven sobre sus elecciones de transición (Punch, 2002).

El enfoque en la agencia nos permite escuchar las voces marginalizadas de las personas jóvenes rurales y enfocarnos en su capacidad de ser y hacer lo que ellos y ellas consideran valioso. Tal como señala Sen (1999), el desarrollo debe ser entendido como un proceso social que amplía la libertad de una persona o que brinda oportunidades a grupos e individuos de mejorar su situación sobre la base de los criterios que ellos valoran. Por esto es importante considerar las percepciones 
locales de bienestar que tienen las personas jóvenes, así como la forma en que estas percepciones influyen en sus capacidades de salir de la pobreza (véase Miyashita, 2009; Steel \& Zoomers, 2009). ¿Qué es para ellas y ellos 'una mejor vida'? ¿Qué hacen ellos para mejorar sus condiciones socioeconómicas y acumular activos claves para transitar hacia la adultez? Una mejor comprensión de los jóvenes, sus transiciones hacia la adultez y la manera en la cual acceden a recursos en sus hogares puede afinar las intervenciones de desarrollo articulándolas a las necesidades concretas de los jóvenes.

Por ejemplo, en los últimos años hemos visto que la promoción del emprendedurismo entre los jóvenes ha adquirido popularidad en los programas de desarrollo. Las microfinanzas para jóvenes se han convertido en una estrategia de desarrollo popular para ofrecer a los jóvenes acceso a activos financieros. Esto implica el desarrollo de productos financieros específicos para jóvenes sobre la base de que ellos y ellas son agentes económicos con necesidad de capital para invertir en estudios, iniciar negocios o comprar una casa (Storm, Porter \& Macaulay, 2010). Sustentado en la experiencia del Youth-Inclusive Financial Services Linkage Program (YFS-Link) se plantea que los servicios financieros juegan un rol importante en el desarrollo de las vidas de las personas jóvenes (2010). Awogbenle (2010) propone la promoción del emprendedurismo como forma de contrarrestar las tasas de desempleo juvenil en países deficitarios como Nigeria. Plantea que las iniciativas emprendedoras son un medio importante para que los jóvenes mejoren sus medios de vida y alcancen la independencia económica. Sin embargo, Bennell (2007, p. 10) afirma que se han encontrado muchos problemas con estos proyecto pilotos de jóvenes. Muchas veces representan una opción solamente para ciertos grupos de jóvenes y además es difícil controlar los créditos de los jóvenes porque son los padres quienes garantizan los créditos y quienes les involucran en las estrategias del hogar más amplio.

De esta forma vemos que en las experiencias de proyectos concretos el enfoque teórico de la agencia raras veces se traduce en un enfoque práctico. Para orientar las intervenciones de desarrollo hacia las realidades de los jóvenes, sus aspiraciones, posibilidades y restricciones todavía necesitamos analizar con mayor detalle la capacidad de los jóvenes de emprender estrategias que les permitan salir a flote (Bucholtz, 2002; Kamete, 2010). Importa en particular saber sus posibilidades de capitalizacion, la interacción con las estrategias del hogar materno y el éxito de sus estrategias. Por esta razón, queremos analizar la posición de las personas jóvenes de la Nicaragua rural en el proceso de desarrollo y sus transiciones hacia la adultez. ¿Cuáles son sus estrategias principales y sus posibilidades de enfrentar situaciones de pobreza mediante rutas que desde su óptica resulten la mejor opción?

\section{Los jóvenes de escasos recursos y sus hogares en la Nicaragua rural}

Muy Muy y Matiguás son municipios atravesados por una vía de pujante producción lechera y cárnica que une Boaco, Muy Muy, Matiguás, Río Blanco y Mulukukú (ver ilustración 2). Sobre esta carretera y sus ramificaciones funciona una red de centros 
de acopio y plantas procesadoras de queso que compiten diariamente por captar la producción láctea de la zona. Estas dinámicas han promovido el incremento de la desigualdad en la zona, quedando marginados grandes segmentos de la población rural (Bastiaensen \& Marchetti, 2010; Flores \& Delmelle, 2006). La mitad de las familias de Matiguás no tiene tierra (INIDE, 2001) y en Muy Muy quienes trabajan principalmente en las cadenas agroindustriales de leche y carne son los 'grandes productores' que tienen más de veinte manzanas, siendo éstos menos de la mitad de la población. Esto implica que la mayoría de la población de ambos municipios, dependiente de actividades agropecuarias, está desventajosamente integrada a estas dinámicas dominantes. Los sin tierra no tienen la posibilidad de involucrarse más que como asalariados informales y quienes tienen pequeñas parcelas no pueden producir lo suficiente como para sacar provecho del mercado de la leche y la carne. Esto se refleja en el hecho de que a pesar de existir una dinamización económica en las cadenas lácteas y cárnicas, la mitad de la población de Muy Muy y Matiguás vive en pobreza extrema (INIDE, 2007).
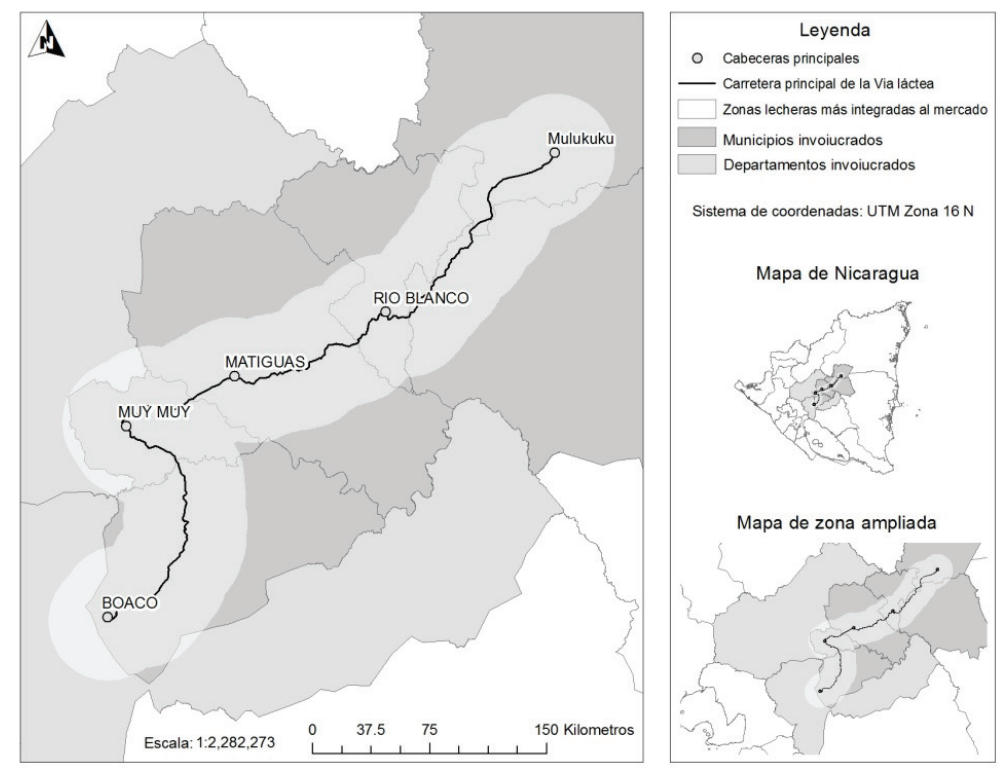

Ilustración 2. Mapa de la vía láctea que conecta Boaco, Muy Muy, Matiguás, Río Blanco y Mulukukú

Fuente: Elaborado por Carlos Sosa, Nitlapan, 2011. Datos del CENAGRO (INIDE, 2001).

Muchos jóvenes rurales en estas zonas están viviendo en situaciones de pobreza y encuentran restricciones para incorporarse a los rubros económicos más dinámicos en el territorio. En la mayor parte de los casos se vinculan con estos rubros más dinámicos mediante los mercados laborales (ordeño, cercado, chapeo de potreros, transporte de leche) y en menor medida como productores de leche a pequeña escala en consorcio con sus progenitores. Tanto El Bálsamo como Pancasán son de 
reciente formación campesina, producto de los procesos de reforma agraria de los ochenta. Están poblados por antiguos asalariados estatales y cooperativistas que han sobrevivido a los ajustes estructurales y los procesos progresivos de reconcentración de la tierra (Alemán, 2010). Hemos observado que la mayoría de los jóvenes en este conglomerado carece de tierra propia. Por lo general los hombres tienen acceso a una o dos manzanas de sus padres. En cambio, pocas mujeres acceden a la tierra o usufructúan la de sus padres. Las que más pueden acceder a la tierra son mujeres 'juntadas', donde en la mayoría de los casos su pareja maneja la parcela.

También en el acceso a otros recursos los jóvenes con quienes hemos trabajado encuentran restricciones importantes. Por ejemplo, pocos jóvenes pueden ahorrar, gastando la mayoría sus ingresos en el sostenimiento del hogar. Quienes ahorran lo destinan principalmente al manejo de riesgos, algunos guardando efectivo en su casa o pasándolo a una persona de confianza. Otros se involucran ocasionalmente en el engorde de cerdos. Por otro lado, hay quienes han recibido crédito para un ciclo agrícola o la compra de ganado mediante micro-financieras o préstamos de familiares; mientras otros han prestado a bancos para viajar Costa Rica. En general, hemos encontrado muchas familias que vivían de préstamo en préstamo, incluso prestando para el consumo del hogar. Estos usos crediticios muestran que varios de sus proyectos económicos son de corto plazo y a veces los jóvenes están endeudándose incluso para comer.

En medio de estas restricciones las personas jóvenes con quienes trabajamos se involucran en una diversidad de actividades agropecuarias y no agropecuarias para asegurar sus medios de vida. Varios hombres jóvenes se incorporan al cultivo de granos básicos, la ganadería de leche y el cultivo de café de sombra, realizándose por lo general en pequeñas parcelas pre-heredadas ${ }^{4}$ o alquiladas. En Pancasán estos hombres tienen más posibilidades de empleo agropecuario estacional, ofreciéndose en haciendas cafetaleras de los alrededores. Algunos además se desenvuelven en labores de carpintería, ocasionalmente en construcción y en acopios de leche y cacao. Las mujeres están más involucradas en el trabajo doméstico (a veces pagado), pulperías y venta de productos caseros (nacatamales, buñuelos, cuajadas) principalmente durante los cortes de café. Ellas se incorporan a actividades de la economía de patio como la crianza de gallinas, engorde de cerdos y cultivo en huertas familiares. Trabajan igualmente en la producción agrícola por lo general como mano de obra familiar o como asalariada temporal en haciendas cafetaleras. Encontramos muchos casos de jóvenes que salen temporalmente a desempeñar labores agrícolas a otras zonas de Nicaragua o a Costa Rica. Por lo general los hombres van a buscar trabajo en la construcción y la agricultura y las mujeres principalmente van a buscar trabajo como domésticas en las ciudades grandes (Steel, Winters \& Sosa, 2011). Estas actividades diversas se imbrican en estrategias de independencia que nosotros mapeamos para entender cómo se despliegan en las rutas de desarrollo de estos jóvenes.

4 En este caso nos referimos a una condición en la cual el padre pone a disposición del hijo o yerno (en su defecto) cierta cantidad de tierra pero no su titularidad. Las mujeres no heredan la tierra, salvo casos excepcionales. 


\section{Las estrategias de acumulación e interdependencia}

Para poder analizar las estrategias de vida de los jóvenes rurales en Muy Muy y Matiguás identificamos tres diferentes grupos de jóvenes en base a la interdependencia que guardan sus estrategias con las de sus padres. El primer grupo está conformado por jóvenes con estrategias integradas con las de sus padres. El segundo grupo está compuesto por jóvenes con estrategias mediadas por los recursos de sus padres. El último grupo son los jóvenes con las estrategias más autónomas. Esta es una agrupación general de las estructuras de los hogares sobre las cuales estos jóvenes transitan hacia la adultez. Conscientes de las limitaciones de encuadrar las dinámicas que los jóvenes implementan en sus hogares, realizamos estas particiones para facilitarnos el análisis de las rutas de desarrollo que siguen los jóvenes, permitiéndonos ver similitudes y contrastes entre los casos e identificar pistas para las intervenciones de desarrollo en el territorio.

\subsection{Estrategias integradas}

El primer grupo, compuesto por once de los veinticuatro casos, lo constituyen jóvenes que continúan viviendo con sus padres o suegros ${ }^{5}$. Este es el grupo más heterogéneo, pues está conformado por jóvenes solteros, parejas jóvenes y madres solteras. En general, estos jóvenes negocian sus estrategias dentro del hogar materno, sosteniéndose estrategias interdependientes. Observamos que es común en estas circunstancias que los jóvenes ya generen ingresos propios bajo el techo del hogar progenitor pero además se suman a la mano de obra familiar. Por ejemplo, Pedro, Juan y Carlos son hombres solteros y 'juntados' que viven con sus padres pero al mismo tiempo trabajan ya sea asalariadamente o en su propio negocio para su bolsa. Además, trabajan la tierra junto con sus padres para la mantención del hogar materno. Aunque hay un caso excepcional donde el padre paga la mano de obra del hijo, la mayor parte de quienes viven en el hogar materno trabajan en la tierra de sus padres sin remuneración monetaria. Por esta razón, analizamos sus estrategias de vida como estrategias integradas con las de sus padres.

En general los jóvenes solteros continúan formando una unidad de producción y consumo con sus padres. Sus actividades económicas forman parte de las estrategias de vida de sus padres, involucrándose en la distribución del trabajo y la manutención del hogar. Sin embargo, estos jóvenes generan ingresos propios para poder desarrollar sus rutas de desarrollo. Por ejemplo, Pedro y Heraldo son una pieza clave de sus hogares pues sus trabajos asalariados representan una entrada significativa para sus hogares, sin las cuales sus padres probablemente se verían en situaciones más precarias. Ellos a su vez han admitido que de los ingresos que generan guardan un parte para sus gastos personales y para poder en algún momento invertir en un negocio propio. Heraldo quiere poner un taller de bicicletas y Pedro está valorando si montar una venta o comprar una vaca.

Las parejas jóvenes que viven con sus padres se maneja en otro nivel de

5 En los casos de parejas, las mujeres por lo general se pasaban a vivir a la casa de los suegros. 
coordinación. Como ya han formado una familia nueva, estos tipos de hogares tienen un centro de decisión emergente, constituido por la pareja joven que les introduce en actividades tanto a lo interno como extra-finca para generar ingresos propios. Sin embargo, al estar adscrita a la estrategia del hogar materno, se involucran en la división del trabajo establecida en este hogar. Esto implica que los hombres por lo general se dividen la coordinación del trabajo en las parcelas y las mujeres en las labores reproductivas. En el caso de los padres o suegros que tienen casa y terreno, el trabajo agropecuario se hace en conjunto con los papás para asegurar el autoconsumo del hogar materno. Algunos incluso pueden hacer trabajo asalariado extra-finca para generar ingresos propios o acceder a crédito con respaldo paterno para poder incrementar el ganado o los cultivos anuales ${ }^{6}$. Las parejas jóvenes que viven con sus padres sin tierra sienten la presión socio-económica de complementar los ingresos del hogar extendido con trabajo asalariado, comercio u ofreciendo servicios. Para generar ingresos que permitan mantener a su familia nuclear y contribuir así a los ingresos del hogar materno, muchos de estos jóvenes salen de la comunidad. Por lo menos hemos conocido que varios hombres salen por temporadas a vender su mano de obra en otros departamentos de Nicaragua o en Costa Rica.

En otras palabras, vivir con los padres representa un reto para la acumulación de recursos propios para vivir aparte, pues se depende mucho del éxito de la estrategia del hogar extendido. Esta situación de dependencia parece ser más sentida por las mujeres. En distintas ocasiones las mujeres juntadas mencionaron el sueño de su propia casa, que se traduce en un sueño de emancipación, pues implica ganar cierta cuota de poder en su núcleo familiar. Por ejemplo, Diana vive en casa de sus suegros junto con su pareja Carlos. Mientras Diana fue enfática en expresar su deseo de moverse a un lugar propio, Carlos parecía más cómodo en la división laboral que tiene con su padre, ya que él se ocupa del ganado y su padre de los cultivos.

Por su parte, las madres solteras que viven con sus padres tienen aún menos posibilidades de independizarse. Sin el apoyo de un compañero para la generación de ingresos y la crianza, ellas tienden a recurrir a sus padres para poder vivir y si se puede, acumular recursos para la manutención de los hijos. Observamos que es común que ellas salgan a trabajar a otros departamentos o a Costa Rica como domésticas o asalariadas agrícolas. En este caso, sus padres constituyen una red social fundamental para las labores de cuido. Hay, por lo general, un fuerte lazo entre la madre que se va y sus padres que se quedan, pues la mama envía remesas al hijo y la familia que le mantiene, contribuyendo así con los gastos del hogar extendido (Steel et al., 2011). Esto se da en un contexto en que el hogar extendido no cuenta con los recursos suficientes para sostener a un nuevo miembro dependiente. En muchos casos la dependencia del cuido de los hijos también se traduce en el hecho de que las madres solteras no puedan trabajar continuamente fuera de la comunidad. Muchas veces las mujeres solteras deben regresar al poco tiempo o al menos siempre que

6 Logramos visualizar que los padres han jugado un papel clave en el acceso y manejo de los créditos. Esto sucede sobre todo en la medida en que dicha inyección de capital se integra a la estrategia del hogar extendido. Así por ejemplo, Carlos y Ana tienen padres en la cooperativa Flor de Pancasán. Gracias a esto pudieron de manera individual acceder a un proyecto de jóvenes emprendedores promovido por la Asociación para la Diversificación y el Desarrollo Agrícola Comunal (ADDAC). Sin el apoyo familiar difícilmente se podrían haber mantenido estas iniciativas empresariales. 
surge una emergencia con su hijo, lo que les impide poder acumular para poder moverse del hogar materno.

En general, el inconveniente de desarrollar actividades económicas integradas a la estrategia del hogar extendido es que se alimenta la interdependencia entre los jóvenes en este grupo y sus padres. Es decir, la salida de los hijos o hijas significaría en parte una pérdida de activos (ganado, tierra o infraestructura) para los padres, pero sobre todo una pérdida de fuerza de trabajo para la estrategia del hogar materno. Por ejemplo, la decisión de Diana y Carlos de moverse del hogar materno hacia una casa y tierra propia significaría para el padre la pérdida de algunas cabezas de ganado que pertenecen a Diana, pero aún más importante, la mano de obra fija de su hijo quien está a cargo del ganado. Y desde la perspectiva del joven, salir sin los recursos suficientes implica también abandonar parte de los activos (casa, tierra y ganado) a los que puede acceder por medio del padre.

Por estas razones, las perspectivas de este primer grupo que convive con sus padres se centran en la posibilidad de acumular suficientes recursos para conseguir un lugar para vivir aparte. Sobre todo para las mujeres 'juntadas', esto significa tener mayor autonomía sobre las rutas de desarrollo que quieren seguir. Aparece como otra perspectiva compartida por los hombres 'juntados' y las madres solteras, la de moverse -al menos por un tiempo- de la comunidad, principalmente para trabajar por salarios más atractivos. Continuar estudios constituye una perspectiva estratégica en este grupo, pues sosteniéndose en los activos de sus padres (casa y tierra) se puede invertir tiempo y recursos en formación. Para quienes siguen estudiando, la probabilidad de escalar en sus estudios recae aparentemente en la posibilidad que tengan ellos mismos de costeárselo vía salario ${ }^{7}$. Sin embargo, esta no es una posibilidad compartida por la mayoría, que carente de recursos e incentivos para invertir en su formación tiende a priorizar la perspectiva de generar más ingresos para eventualmente dejar el nido materno.

\subsection{Estrategias mediadas por los padres}

El segundo grupo cuenta con nueve casos y está conformado por hogares jóvenes viviendo en casa aparte, pero en terreno de un familiar. Estos hogares los constituyen principalmente parejas jóvenes que están intentando construirse una vida independiente sin desvincularse de las estrategias socioeconómicas de sus padres. Esto dependerá de los recursos disponibles de los padres o suegros y el nivel de colaboración que mantengan. Por ejemplo, en muchos casos los padres son 'mediadores de recursos' para estos hogares jóvenes, abriéndoles puertas para financiamiento y redes sociales. Dicha mediación está relacionada a la interdependencia prevaleciente entre las estrategias de vida del hogar joven y el de sus padres, a pesar de no vivir bajo el mismo techo. Los jóvenes aún tienen que negociar sus rutas de desarrollo con sus padres, ya que éstos no dejan de ser clave en

7 Observamos que quienes han tenido esta experiencia previamente se enfrentan a la problemática de no poder pagar estudios superiores con sus bajos salarios, lo que al regresar al territorio restringe sus oportunidades laborales (Krauskopf, 1998 citado por Wolseth \& Babb, 2008). Como Sayra explica: "El trabajo de doméstica te da para estudiar secundaria pero no alcanza para la universidad, es demasiado poco lo que se gana”. 
el alcance de metas relevantes para la estrategia del nuevo hogar joven. A su vez, el hogar materno estará interesado en seguir manteniendo la fuerza de trabajo de los hijos en su unidad de producción.

Una modalidad en que los padres funcionan como mediadores es cuando éstos forman parte de una organización y los jóvenes pueden acceder a ciertos beneficios por ser hijos de los asociados. Por ejemplo, Ileana y Alfredo son jóvenes 'juntados' que tuvieron la oportunidad de participar en el proyecto de jóvenes emprendedores promovido por ADDAC gracias a que sus padres pertenecen a la cooperativa Flor de Pancasán. A diferencia de los casos de Ana y Carlos (del primer grupo) que acceden a activos financieros capitalizados por el hogar materno, los créditos a los que acceden Ileana y Alfredo no se integran a la estrategia del hogar materno sino que son activos mediados por éste para la estrategia del hogar joven.

Encontramos que en estos hogares jóvenes la ruta agropecuaria (estrategias de vida basadas en actividades agrícolas y pecuarias) ocupa un espacio importante en sus estrategias de vida a diferencia del primer grupo, en donde en muchas ocasiones las rutas están menos explícitamente vinculadas a lo agropecuario. Quienes priman las actividades agropecuarias en sus estrategias de vida mantienen un vínculo de interdependencia con sus padres sostenido en gran parte por acuerdos de preherencia, los que hemos explicado en el apartado anterior. Ésta también resulta en una modalidad de 'mediación de recursos' que forma parte tanto de la estrategia del hogar materno como del hogar joven. El padre, al ofrecer tierra en pre-herencia asegura que el hijo no se separe completamente de la estrategia de sus padres, sobre todo porque representa una mano de obra comprometida, que puede resultar costosa vía mercado en la zona rural. El hijo, por su lado, se asegura el acceso al activo tierra -y por tanto indirectamente a productos agrícolas para el autoconsumo-, con las posibilidades de que le sea heredada. De esta forma, en varios casos continúan trabajando sin salario la tierra de sus padres, como compromiso (explícito o implícito) por esta mediación de recursos de sus padres.

Dentro de las posibilidades que ofrecen las pequeñas parcelas en pre-herencia ${ }^{8}$, la estrategia de estos hogares jóvenes es poder diversificar los cultivos por épocas. Por lo general cultivan granos básicos combinándolos con cultivos más dirigidos a la venta, como hortalizas, tubérculos y frutas. Quienes se dedican solamente a la agricultura alquilan tierra adicional, mientras otros complementan con trabajo asalariado, dentro o fuera de la comunidad, en tiempos muertos ${ }^{9}$. En ambos casos se trabaja también con el ganado o con los cultivos de los padres. No obstante, enfrentan las tensiones de pre-herencia al sentir la inseguridad por la tenencia sobre la inversión en cultivos de alta renta. Siendo las tierras de sus familiares, les preocupa no tener certeza sobre la recuperación de las inversiones que hagan en éstas. Por ejemplo, Mayra y Alfredo, viendo las oportunidades de mercado del café en la zona, han comprado las semillas para sembrar café en tierras de un tío. Normalmente la primera cosecha con la que ellos podrían recuperar la inversión sería en cuatro años, lo que les hace dudar sobre la conveniencia de esta inversión en una tierra

8 Por lo general, los jóvenes pre-heredan entre 0.5 y 2 manzanas dependiendo de la disponibilidad de tierra del padre.

9 Tiempo en que no hay actividad agrícola cuando se trabaja con sistemas dependientes de las estaciones. Éstos por lo general se dan entre finales de febrero y de abril. 
que no les pertenece. Por otra parte, dichas estrategias interdependientes no están ausentes de tensiones e inequidades. A veces, el acuerdo de trabajo sin salario por parte de los hijos puede implicar una carga de trabajo que no permite dar el salto a los hogares jóvenes que desean acumular recursos para alcanzar su independencia socioeconómica. Por tanto, lo que ocurre en ocasiones es que el hogar joven puede sostenerse con la tierra pre-heredada y los ingresos complementarios pero no siempre tiene las posibilidades de acumular lo suficiente para alcanzar sus objetivos de tener una casa y terreno propio.

Logramos observar, a su vez, que estos acuerdos de pre-herencia se dan predominantemente entre padres e hijos. Las mujeres parecen no tener derecho a heredar bajo el argumento de que ellas no trabajan la tierra. De esta forma se oculta el papel que juegan ellas en la estrategia del joven hogar, donde ellas se suman a la mano de obra familiar en la parcela. Además, ellas buscan cualquier oficio para poder ganarse algún dinero extra para contribuir al sustento del hogar. Ellas van a cortar café, venden gallinas ocasionalmente, engordan cerdos o venden productos caseros como buñuelos, pan, nacatamales, etc. De acuerdo con lo que ellas comentan, todas sus ventas tienen auge principalmente en la época de corte de café porque en general la gente tiene más dinero para comprar. Sin embargo, esto no significa que ellas no sean sujetas de acceso a otros activos. En diferentes casos ellas habían logrado obtener animales -como vacas, cerdos y gallinas -, equipos o capital financiero para montar un negocio, por medio de sus familiares o incluso de fuentes externas como proyectos de desarrollo.

Otro elemento característico de este grupo que vive aparte es que sus viviendas son comúnmente pequeñas estructuras de tabla que han logrado construir con el apoyo de familiares y del trabajo asalariado. Estas casas son vistas como 'temporales', siendo fáciles de desmontar y remontar en otro lado. Pudimos averiguar que la condición temporal de estas casas no sólo responde a una evidente limitante de recursos para edificar unas más estables, sino también a la estrategia misma del hogar en condiciones transitorias. Efectivamente, para estos jóvenes, mientras vivan en tierra ajena y exista la posibilidad e intensión de moverse a otras tierras, difícilmente podrán invertir en la estabilización de su vivienda actual. Esto coincide con la perspectiva compartida en este grupo de poder tener su propio terreno donde hacer su casa.

Las aspiraciones de estos hogares jóvenes es acumular por medio de la actividad agropecuaria. Por lo menos una parte de sus planes se articula alrededor de conseguir tierra propia. No obstante, vale mencionar que en el caso de las mujeres hay una perspectiva de involucrarse en nuevas actividades de comercio y servicios o mejorar el que tienen -ya sea pulpería, repostería o costura. Lo que sí es compartido por estos hogares jóvenes es el plan de construirse una casa más firme y acceder a capital de inversión para sus proyectos. Uno de estos proyectos importantes es formar a sus hijos para que amplíen sus oportunidades laborales y que al crecer les retribuyan este apoyo. De tal manera, los hijos de estos jóvenes ya entran a jugar un papel, aunque a largo plazo, dentro de la estrategia de vida de su joven hogar. Esto implica invertir en la educación básica de sus hijos actualmente y acumular recursos para invertir en su educación superior. 


\subsection{Estrategias de mayor autonomía}

El tercer grupo, compuesto sólo por cuatro casos, lo conforman jóvenes que viven en terreno y casa propia. El hecho de que sean pocos casos nos muestra lo complejo que es para muchos jóvenes rurales alcanzar la meta de tener una casa y terreno propios, pero sobre todo, lo difícil que resulta transitar hacia la adultez y ganar independencia. Siendo personas mayores de 27 años, este es el grupo más independiente, viviendo en casas más estables y en algunos casos de concreto. Aunque son sólo cuatro casos, las edades en las que rondan nos dicen que para lograr su independencia socioeconómica los jóvenes tienen que pasar por un arduo proceso de idas y venidas. Sin embrago, cada caso tuvo su particularidad en el proceso de lograr conseguirse un terreno y casa propia para vivir.

Guillermina, por ejemplo, es el caso excepcional de una mujer soltera que se fue nueve años a trabajar a Costa Rica como doméstica y luego regresó a su comunidad. Ha juntado dinero con su hermana menor para comprarse un terreno y una casa para ellas, sus hermanos y sus padres. La otra joven que regresa de Costa Rica es Jesenia. Ella, su esposo e hija salieron a Costa Rica donde vivieron por cinco años donde un hacendado al que le trabajó su marido. El salario devengado les permitió ahorrar e invertir sus ahorros de vuelta en la comunidad de origen. Su experiencia es una historia poco común que constituye el logro al que las familias jóvenes de la zona aspiran y muchas veces fracasan en el intento de alcanzar. Esto significa tener la posibilidad de salir de la comunidad para acumular los recursos necesarios que les permitan hacerse una vida al retornar (Steel et al., 2011).

Los otros dos casos son madres solteras que pudieron aprovechar el apoyo puntual de sus padres y de fuentes externas para lograr tener un lugar propio para vivir. Sus experiencias no sólo representan el aprovechamiento de las oportunidades para adquirir una vivienda o terreno, sino a su vez la confluencia del apoyo efectivo de sus padres al mediar recursos. En el caso de Isidora, la herencia de una vaca fue un activo importante para poder comprar un terreno en un momento en que estaba barato. Mientras Ligia, para acceder a un proyecto habitacional auspiciado por DESWOS ${ }^{10}$ y ejecutado por el Grupo Sofonías ${ }^{11}$, logra conseguir una manzana de tierra de sus padres para poder sembrarla y mantenerla con árboles maderables, y así cumplir con el requisito que la vuelve beneficiaria de su actual vivienda.

Entonces lo interesante de este grupo es que el salto dado hacia el alcance de mayor independencia ha dependido no sólo de actividades agropecuarias. Dos de los casos han logrado acumular recursos para independizarse trabajando asalariadamente varios años en Costa Rica, mientras los otros dos casos son madres solteras que han aprovechado el acceso a activos claves gracias al manejo de sus redes familiares, por medio de las cuales han fluctuado activos como tierra y ganado para poder tener una casa y terreno propios.

Las perspectivas de este grupo de jóvenes apuntan, por un lado, a la persecución de mejores oportunidades laborales, considerándose opciones como salir del país a trabajar asalariadamente o que el marido encuentre trabajo asalariado

10 Deutsche Entwicklungshilfe für soziales Wohnungs- und Siedlungswesen (Ayuda Alemana para el Desarrollo de Viviendas y Asentamientos Sociales)

11 El Grupo Sofonías es una ONG nacional ubicada en Diriamba, Carazo. Durante el año 2003 ejecutó un proyecto de construcción de viviendas en Matiguás priorizando a madres solteras. 
en la zona, como sucede con Jesenia. Por otro lado, apuntan a la adquisición de activos para emprender una actividad propia como la ganadería o la apertura de pequeños negocios de panadería y pulpería. La meta más importante aquí consiste en generar más ingresos para la manutención y educación de sus hijos. En este sentido, muchas de las aspiraciones de estos hogares jóvenes recaen en las hijas e hijos, particularmente en la posibilidad de que éstos se profesionalicen para tener más oportunidades laborales que puedan ser retribuidas.

Por último, es válido mencionar que observamos un cambio importante en el ciclo de vida de estos jóvenes mayores. A pesar de que mantienen algún vínculo socioeconómico con sus padres, ahora estos jóvenes comienzan a construir estrategias interdependientes con sus propios hijos. Los hijos e hijas mayores de Isidora y de Ligia están generando ingresos mediante trabajo asalariado y apoyando la producción familiar. En el caso de Ligia, quien vive con sus cinco hijos, su hijo mayor, de 17 años, se responsabiliza junto con ella de la producción de granos básicos de autoconsumo. Él estudia en secundaria y también trabaja en las plantaciones de su abuelo como mano de obra familiar, volviéndose así un hijo potencialmente heredable. De esta forma, las estrategias del hogar materno y del hogar de Ligia se mantienen vinculadas. Por su parte, Isidora quien tiene una hija de 18 años y uno de 10, depende en buena medida del trabajo asalariado de su hija, quien es también madre soltera y trabaja como doméstica en Managua enviando remesas a Isidora por cuidar al nieto. La estrategia de vida del hogar de Isidora se sostiene sobre el vínculo de colaboración entre ella y su hija. De esta forma podemos observar un ciclo repetitivo en las estrategias de vida de estos jóvenes, pues los hijos de los jóvenes en este grupo están en la misma posición de los jóvenes en el primer grupo, pues sus estrategias de vida están integradas con las de sus padres.

\section{Conclusiones}

En este artículo hemos analizado las estrategias de vida y las rutas de desarrollo de jóvenes rurales en Muy Muy y Matiguás con la intención de poder formular algunas recomendaciones para las intervenciones de desarrollo con jóvenes. La primera conclusión importante es que los jóvenes rurales resultan ser un grupo heterogéneo. Las personas jóvenes no pueden ser clasificadas únicamente sobre la base de su edad, pues también hay diferencias de género, de medios y estrategias de vida, así como de fases en el ciclo de la vida.

El acceso a recursos es, por ejemplo, claramente diferenciado entre hombres y mujeres. Los hombres con oportunidades de usufructuar tierra de familiares tienen más posibilidades de acceder a otros capitales como activos financieros para la producción. Las mujeres jóvenes, contrariamente, al no pre-heredar ni recibir tierra para usufructo, reducen su oportunidades de acceso a otros activos. La edad también tiene influencia en el nivel de autonomía y dependencia que pueden tener los jóvenes con sus padres. Aunque el vínculo con las estrategias de vida de los padres está siempre presente, hemos observado que sólo los jóvenes mayores constituyen el grupo más independiente, teniendo estrategias más autónomas frente a sus padres y viviendo en casas independientes y más estables. Las estrategias de vida de los demás jóvenes se perfilan en medio de 'interdependencias negociadas' (Punch, 2002) 
donde sus estrategias de vida están integradas con las del hogar materno o mediadas por los recursos de los padres.

Este énfasis que hacemos en la heterogeneidad de los jóvenes quiere llamar la atención sobre la necesidad de reconocer las historias personales de los jóvenes, sus posibilidades y limitaciones en la búsqueda de mejores oportunidades de vida. Si queremos mejorar las estrategias de desarrollo con jóvenes es importante identificar estas diferencias pues nos indican que no todos los jóvenes que viven en el medio rural comparten las mismas necesidades, aspiraciones y percepciones. Por ejemplo, reconocer que las estrategias de las personas jóvenes varían según el nivel de interdependencia que mantienen con la estrategia del hogar materno implica que sus demandas de activos y perspectivas dependerán del nivel de interdependencia en que se encuentren.

A su vez, esta identificación de heterogeneidades nos permite visualizar cómo el alcance de ciertas metas en las estrategias de vida de los jóvenes -como acceder a casa y tierra propia- es posible según distintas rutas de desarrollo. Las rutas de los hogares jóvenes son mixtas, es decir que no están solamente vinculadas a actividades agropecuarias. Las actividades asalariadas y no agropecuarias también juegan un papel clave en las estrategias de los jóvenes que vimos en los diferentes grupos.

En este sentido, la 'movilidad' ha mostrado ser un activo mediante el cual los jóvenes pueden acceder a recursos en su transición hacia la independencia. Con movilidad nos referimos a las salidas que hacen los jóvenes de sus territorios para acceder a recursos mediante trabajo asalariado. Dentro de quienes implementan estrategias integradas con sus padres, la movilidad es comúnmente usada como puente entre el hogar materno y la posibilidad de construirse un propio hogar aparte, tal como sucede con las madres solteras de este grupo (Steel et al., 2011). Para quienes gozan de la mediación de sus padres o quienes tienen estrategias más autónomas, el moverse puede servir para múltiples propósitos dentro del hogar joven. Moviéndose de la comunidad unos pueden pagar deudas, otros logran mantener a sus hijos, e incluso algunos pueden acumular capital para invertir en tierra o negocios no agropecuarios.

Sobre la base de estas estrategias de vida, consideramos a los jóvenes como agentes activos en el proceso de desarrollo. Los jóvenes y sus hogares no actúan de forma aislada para enfrentar las restricciones y aprovechar las oportunidades que el medio les provee. Por el contrario, se articulan con sus redes, sobre todo familiares, ya sea para echar a andar una empresa conjunta, para conseguir capital financiero, para labores de cuido o para resolver emergencias. Pero al mismo tiempo los jóvenes rurales son actores que ya están involucrados en varias iniciativas propias para tratar de mejorar su situación. Las intervenciones de desarrollo presentes en la zona deberían partir del fortalecimiento de estas varias iniciativas en que los jóvenes ya están involucrados. Desde nuestra perspectiva, al menos correspondería considerar las estrategias móviles de las personas jóvenes en cualquier plan o proyecto de desarrollo que considere trabajar con jóvenes. Esta observación intenta responder al hecho de que los jóvenes muchas veces tienen un interés en proyectos pequeños que presenten resultados a corto plazo, pues proyectos de largo plazo pueden restringir su movilidad, lo que significa correr el riesgo de bloquear un recurso importante para sus estrategias de vida. 
En este sentido, es preciso recalcar que la vida de los jóvenes mismos está aun en movimiento, sobre todo cuando no tienen un lugar fijo donde vivir. Además tenemos que reconocer a los jóvenes como agentes económicos con necesidades de financiamiento para inversiones claves (casa, tierra, activos líquidos, etc.) que pueden permitirles dar saltos en sus procesos de transición hacia la adultez. En este cometido, la promoción del emprendedurimo como iniciativa de financiamiento tiene una limitada posibilidad de difusión. Por un lado debido a que no todos los jóvenes quieren formar empresas y por otro lado, a que en el contexto rural la demanda de servicios es escasa. Por tanto, para jóvenes que están orientándose por rutas más agropecuarias y cuentan con algunas posibilidades como 'acuerdos de palabra' sobre tierra en pre-herencia o arrendada, habría que experimentar con algunas modalidades de formalización de acuerdos de pre-herencia o arriendos a largo plazo que puedan generar cierta seguridad sobre la tenencia y estimular la inversión.

\section{Referencias bibliográficas}

Alemán, M. (2010). Borrador de discusión sobre Territorio de Matiguás. Managua. Manuscrito no publicado.

Ansell, N. (2004). Secondary schooling and rural youth transitions in Lesotho and Zimbabwe. Youth and Society, IV (32), 183-202.

Awogbenle, A. C. (2010). Youth unemployment: Entrepreneurship development programme as an intervention mechanism. African Journal od Businness Management, 4, 831-835.

Bastiaensen, J., \& Marchetti, P. (2010). Microfinanzas rurales y cadenas de valor agropecuarias. IOB. Antwerp: Institute of Development Policy and Management.

Bastiaensen, J., De Herdt, T. \& D’Exelle, B. (2005). Poverty Reduction as a Local Institutional Process. World Development, VI (33), 979-993.

Bennell, P. (2007). Promoting Livelihood Opportunities For Rural Youth. Working paper. Recuperado el 30 de junio de 2011, de http://www.ifad.org/events/gc/30/ roundtable/youth/benell.pdf

Bucholtz, M. (2002). Youth and Cultural Practice. Annual Review of Anthropology (31), 525-552.

Caputo, V. (1995). Anthropologies silent others - consideration of some conceptual and methodological issues for the study of youth and children's cultures. London: Routledge.

Carney, D. (1998). Sustainable rural livelihoods: what contribution can we make? London: DFID.

Chambers, R. (1995). Poverty and Livelihoods: Whose Reality Counts? Environment and Urbanization, I (7), 173-204.

Chant, S. \& Jones, G.A. (2005). Youth, Gender and Livelihoods in West Africa: perspectives from Ghana and The Gambia. Children's Geographies, 3 (2), 185 199.

Collier, J. \& Collier, M. (1996). Visual Anthropology. Photography as a Research Method. Albuquerque: University of New Mexico Press.

Crivello, G. (2010). 'Becoming somebody': youth transitions through education and 
migration in Peru. Journal of Youth Studies , IV (14), 395-411.

Flores, S. \& Delmelle, G. (2006). Detailed Market Chain Analysis for Smallholders Affected by the CAFTA Agreement in Nicaragua. IFPRI. Managua: Nitlapan.

Giddens, A. (1984). The constitution of Society; Outline of the Theory of Structuration. Cambridge: Polity Press.

INIDE. (2007). Perfil y características de los pobres en Nicaragua 2005 (Encuesta de Hogares sobre Medición de Nivel de Vida 2005). Managua: INIDE.

INIDE. (2001). Tercer Censo Nacional Agropecuario. Managua: INIDE.

James, A. \& Prout, A. (1990). Constructing and reconstructing Childhood: Contemporary Issues in The Sociological Study of Childhood. The Falmer Press .

Jeffrey, C. \& Mcdowell, L. (2004). Youth in a Comparative Perspective: Global Change, Local Lives. Youth E⿱ Society, 131-142.

Kaag, M., De Haan, L., de Bruijn, M., van Dijk, J. W. M., van Berkel, R., Nooteboom, G., Zoomers, A. \& Hoordijk, M. (2004). Ways forward in livelihood research. En D. Kalb, W. Pantsers, \& H. Siebers (Eds.). Globalization Development Themes and Concepts in Current Research (pp. 49-74). Dordrecht, Boston, London: Kuwer Academic Publishers.

Kamete, A. Y. (2010). Defending Illicit Livelihoods:Youth Resistance in Harare's Contested Spaces. International Journal of Urban and Regional Research, 34 (1), $55-75$.

Liebel, M. (2003). Working children as social subjects: the contribution of working children's organizations to social transformations. Childhood, III (10), 265 285.

Lloyd, C. (2005). Growing up global the changing transitions to adulthood in Developing Countries. (N. R. academies, Ed.) Washington D.C.: The national academies press.

Long, N. (2001). Development Sociology: actor perspective. London: Routledge.

Miyashita, A. (2009). Killing the Snake of Poverty: Local Perceptions of poverty and Wellbeing and People's Capabilities to Improve their Lives in the Southern Andes of Peru. Amsterdam: Dutch University Press.

Punch, S. (2002). Youth transitions and interdependent adult-child relations in rural Bolivia. Journal of Rural Studies (18), 123-133.

Sen, A. (1999). Development as Freedom. Oxford: Oxford University Press.

Steel, G., Winters, N. \& Sosa, C. (2011). Mobility, translocal development and the shaping of development corridors in (semi-)rural Nicaragua, International Development Planning Review, Special Issue forthcoming November 2011.

Steel, G., \& Zoomers, A. (2009). Social mobility in the Central Andes of Peru and Bolivia: towards a more dynamic and multi-local vision of poverty alleviation. International Development Planning Review, IV (31 ), 377-398.

Storm, L., Porter, B. \& Macaulay, F. (2010). Emerging guidelines for linking youth to financial services. Enterprise Development and Microfinance, 21 (4), 307-323.

Valentine, G. (1996). Angels and devils: moral landscapes of child-hood. Environment and Planning D: Society and Space (14), 581-599.

Van Blerk, L. (2008). Poverty, migration and sex work: youth transitions in Ethiopia. Area, 245-253.

Wolseth, J., \& Babb, F. E. (2008). Youth and Cultural Politics in Latin America. Latin American Perspective Issue, 35 (4), 3-14. 